\title{
eJRIEPS
}

Ejournal de la recherche sur l'intervention en éducation physique et sport

$17 \mid 2009$

Varia

\section{La « zone de rencontre » en Education Physique et Sportive : lieu d'interaction, de construction et de transmission des savoirs}

Nathalie Baeza, Deborah Nourrit-Lucas et Daniel Bouthier

\section{(2) OpenEdition}

Édition électronique

URL : http://journals.openedition.org/ejrieps/5571

DOI : 10.4000/ejrieps.5571

ISSN : 2105-0821

Éditeur

ELLIADD

Référence électronique

Nathalie Baeza, Deborah Nourrit-Lucas et Daniel Bouthier, « La « zone de rencontre » en Education Physique et Sportive : lieu d'interaction, de construction et de transmission des savoirs », eJRIEPS [En ligne], 17 | 2009, mis en ligne le 01 avril 2009, consulté le 18 mars 2021. URL : http:// journals.openedition.org/ejrieps/5571 ; DOI : https://doi.org/10.4000/ejrieps.5571

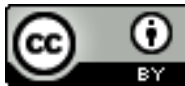

La revue eJRIEPS est mise à disposition selon les termes de la Creative Commons Attribution 4.0 International License. 
La «zone de rencontre » en Education Physique et Sportive : lieu d'interaction, de construction et de transmission des savoirs.

Nathalie Baeza*, Deborah Nourrit-Lucas*, \& Daniel Bouthier**

* Faculté du Sport et de l'EP, 2, allée du Château, BP 6237, 45062 ORLEANS Cedex 2. Laboratoire AMCO, Université Orléans.

** IUFM d'Aquitaine-Université Bordeaux IV, Laboratoire LACES EA4140, Bordeaux 2.

Résumé

L'objectif de cet article est d'étudier, à l'intérieur de rites « d'interaction » (Goffman, 1974), l'espace de rencontre entre l'enseignant d'EPS et ses élèves.

Notre inscription épistémologique est plurielle. Elle s'articule autour des modèles microsociologique et interactionniste de la classe ainsi que de la didactique professionnelle.

Une triangulation des données est utilisée afin de construire une "validité locale et contextuelle ». Ainsi, des questionnaires, des observations de type ethnographique et une étude de cas empruntée au cadre de l'ergonomie de l'activité font émerger l'existence d'une "zone de encontre », espace interactionnel, créé dans l'action, entre l'enseignant d'Education Physique et Sportive et ses élèves.

Cette "zone de rencontre " met en évidence l'existence d'une relation didactique où se joue d'une part la construction de l'identité enseignante et d'autre part la transmission des savoirs.

L'objectif de cet article est d'étudier l'espace de rencontre en Education Physique et Sportive (EPS) ainsi que l'organisation du milieu pendant ces phases dites d'interaction entre l'enseignant et ses élèves. Nous tentons de développer ici une approche originale pour décrire ces moments. Nous postulons qu'ils s'organisent autour de « rites d'interaction » (Goffman, 1974) et qu'ils participent de la transmission des savoirs selon différentes focales. L'étude des interactions en " face à face » dans les cadres naturels est rarement mise en avant dans les recherches sur l'enseignement de l'EPS. Pourtant, l'objet à étudier se laisse identifier : il s'agit de cette classe d'évènements qui ont lieu lors d'une présence conjointe et en vertu de cette présence conjointe. En didactique, AmadeEscot (1996) parle d'activité professorale en situation concrète, c'est-à-dire de 
l'organisation et de la régulation de l'activité des acteurs dans les espaces interactionnels. Ces derniers se construisent à l'intérieur des situations d'enseignement.

Les observations et les analyses de ces rencontres interactionnelles favorisent l'émergence d'interactions didactiques constitutives de ce qui est couramment appelé « l'enseignement » et «l'apprentissage » (Amade-Escot, 2002). Nous étudions plus particulièrement la construction de l'espace interactionnel du point de vue de l'enseignant et nous décrivons les unités d'interaction naturelles qui s'élaborent. Nous appelons cet espace "zone de rencontre ».

II s'agit ainsi d'appréhender le rôle des interactions locales dans une perspective de didactique professionnelle, dans un contexte humain et physique, complexe et dynamique, dans son caractère singulier et générique. De ce fait, nous décrivons l'activité (Bouthier et al., 1995) des acteurs dans les interactions et plus particulièrement dans la « zone de rencontre ». Celle-ci existe dans un contexte spatio-temporel spécifique : celui de la leçon d'EPS.

Les interactions sont appréhendées comme des modes d'adaptation de l'action de l'enseignant et comme une composante de l'action didactique. Les interactions locales deviennent un véritable objet d'étude.

\section{Cadre théorique}

Notre inscription épistémologique s'appuie sur trois sciences de l'action :

1. 1. Le modèle microsociologique

La perspective adoptée par Goffman est héritière d'un courant de pensée préoccupé par le problème de la réalité. Situé à l'interface de deux paradigmes dans le domaine des sciences sociales (ethnométhodologie et interactionnisme symbolique), l'auteur opère en cherchant à décrire les circonstances qui conditionnent « la perception de la réalité », les situations vécues, les convictions, les engagements qui engendrent «l'impression de réalité » (Marcelli \& Milani, 1999).

Goffman (1974) met en avant la notion de rites d'interaction à partir d'une approche tout à fait particulière, dans le sens où, d'une certaine manière, il l'a extrait des références au magique et au sacré pour l'utiliser dans la lecture du quotidien. II se propose de poursuivre un double objectif : décrire les unités d'interaction naturelles qui s'élaborent dans le cadre du quotidien et révéler l'ordre normatif qui prévaut dans et entre ces unités, c'est-à-dire l'ordre comportemental qui existe en tout lieu fréquenté par des hommes. Ces rites permettent à la fois la construction de soi et la possibilité d'une vie sociale dans la classe. Pour reprendre une opposition introduite par l'auteur, les phénomènes relèvent moins d'un 
ordre social que de l'ordre de l'interaction, moins de la structure de la vie sociale que de la structure de l'expérience individuelle de la vie sociale. L'analyse des procédures, par lesquelles les acteurs sociaux s'accordent entre eux, montre qu'ils savent précisément reconnaître et juger des situations pour définir les conduites appropriées.

Autrement dit, l'expérience sociale ne s'organise pas seulement selon l'ordre des identités et des statuts mais selon un répertoire de situations qui a son vocabulaire et son déterminisme, son espace de contraintes et de négociation. Ces différents concepts construits en dehors du champ des recherches en éducation semblent pertinents pour analyser les situations de classe en EPS.

Ainsi, un nouvel objet est introduit : la situation d'interaction qui demande cependant une certaine vigilance épistémologique.

1. 2. Le modèle interactionniste du système classe

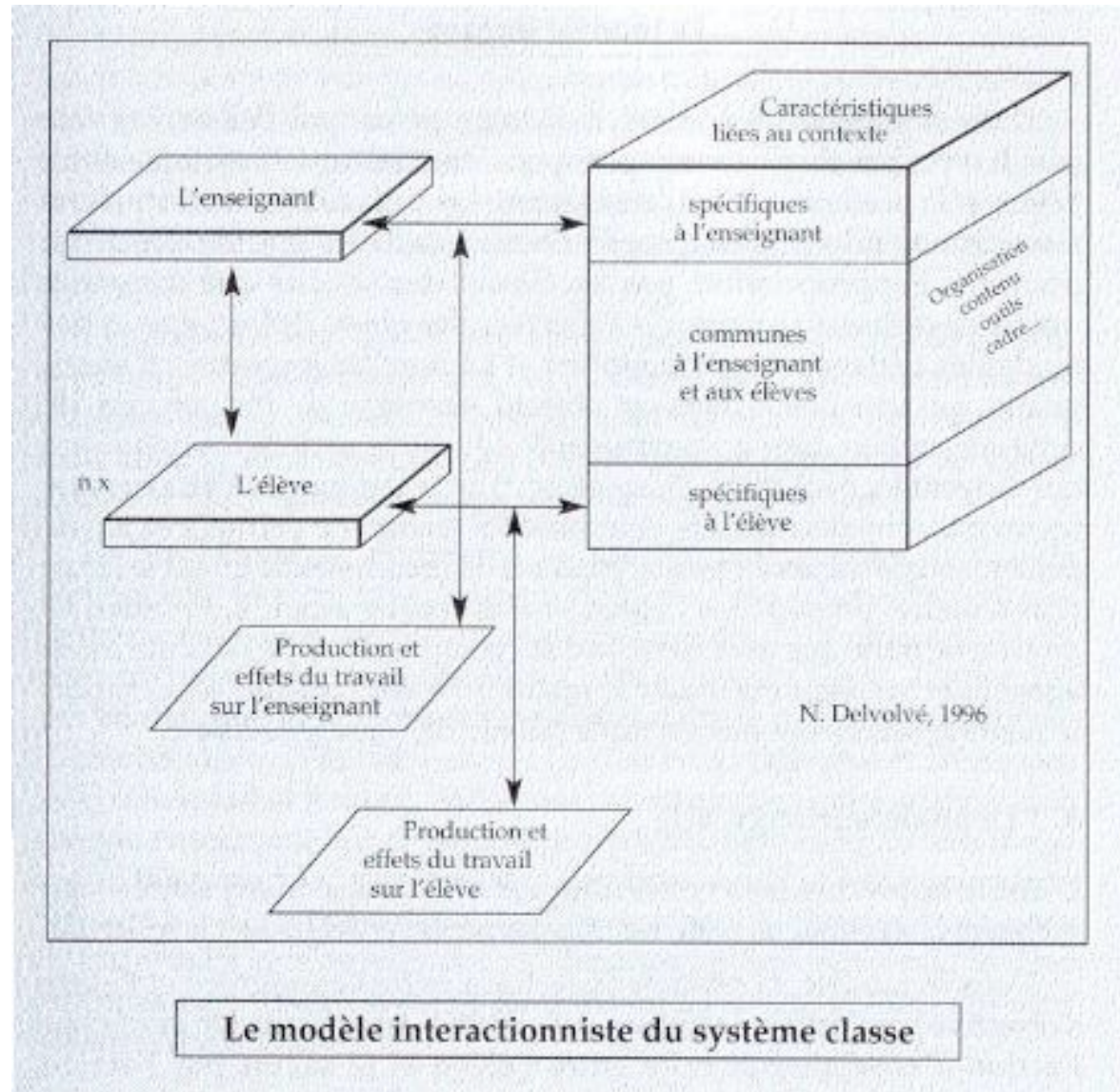

Figure 1. Le modèle interactionniste du système classe. Delvolvé \& Margot (1996)

Dans ce modèle, l'enseignant joue un rôle particulier qui se réfère à une situation caractérisée par un contexte social et culturel, par des composantes de situations (Delvolvé, Margot, 2001) qui sont réfléchies du point de vue des finalités : 
- prise en compte des paramètres contextuels, humains, techniques, organisationnels dans l'analyse de ce que fait et subit un individu à un moment donné ;

- rôle des facteurs de contexte sur l'apprentissage y compris les interactions sociales (la réalité sociale dans laquelle se construit la connaissance).

\section{3. La didactique professionnelle}

Elle a pour but d'analyser le travail en vue de la formation des compétences professionnelles (Pastré, Mayen \& Vergnaud, 2006). Elle aide également à comprendre l'agir professionnel (Samurçay \& Pastré, 1998), c'est-à-dire à analyser l'activité réalisée par l'enseignant en situation de travail, d'un point de vue objectif et subjectif. On ne peut pas comprendre l'activité de l'acteur si on n'a pas une connaissance minimale de la situation et de ses caractéristiques. II s'agit de concevoir des situations de formation ou d'enseignement dites « ergonomiques » pour permettre à un maximum d'élèves de s'approprier les savoirs. En résumé, le cadre d'analyse de la «zone de rencontre » s'articule à partir d'un modèle de type interactionniste où la situation et l'activité sont en interaction. De même, décrire et analyser l'activité de l'enseignant d'EPS, c'est postuler que son intervention existe à travers un emboîtement de strates qui pour certaines sont prévisibles car socialement construites et ritualisées et pour d'autres s'élaborent dans l'urgence de la rencontre. Ainsi, il est possible de distinguer trois strates d'analyse (Figure 2):

- la macro structure sociale dans laquelle l'enseignant a des missions d'instruction, d'éducation et de formation

- la méso structure interactionnelle où l'enseignant ajuste sa démarche au regard de ses élèves. Nous signalerons la présence du contrat didactique (Brousseau, 1978) et nous retiendrons l'idée de partage des responsabilités, ainsi que la présence d'un espace de dialogues (Jonnaert, 1996).

- la micro structure interactionnelle, lieu de structuration de soi et de transmission des savoirs. 


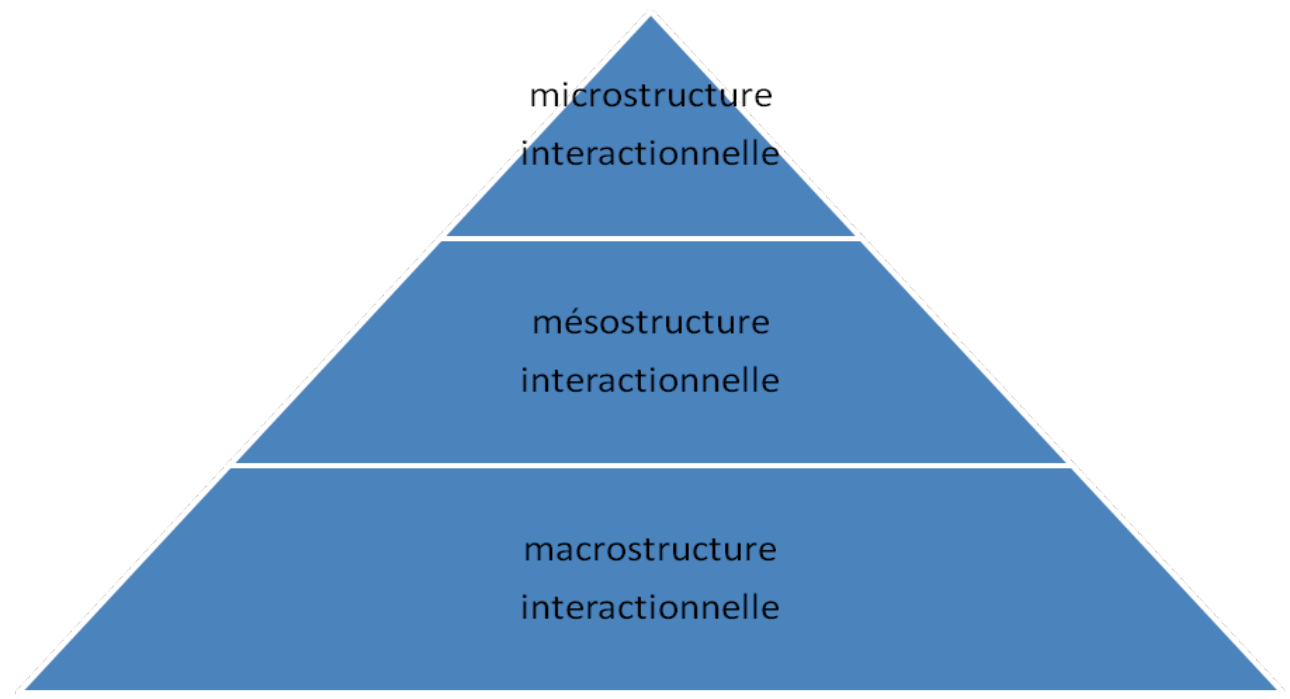

Figure 2. Les strates d'analyses. (Pastré, Samurçay, Bouthier, 1995)

\section{Méthode}

Elle s'articule à partir de trois analyses qualitatives. Notre objectif est de rechercher une unité des catégories d'analyse pour donner une cohérence à l'ensemble des données empiriques.

\section{1. Le questionnaire}

II est structuré en trois phases relatives aux trois rites d'interaction retenus pour définir le cadre de la leçon d'EPS:

le rite de présentation (RP): «lorsque vous présentez une leçon» qui se caractérise par les actes spécifiques par lesquels l'enseignant fait savoir aux élèves comment il les considère et comment il les traitera au cours de l'interaction à venir : c'est la prise en main et la présentation de la leçon. Ce rite marque le rapprochement ou l'éloignement entre l'enseignant et les élèves. Le rite de confirmation (RC): «lors des situations d'apprentissage où les élèves travaillent par groupes, par ateliers et où vous interagissez directement avec ces derniers en particulier ou avec des petits groupes». Ici, il s'agit pour l'enseignant de réaffirmer l'image que l'on souhaite donner ainsi que l'attention et l'intérêt que l'on porte à autrui. Le face à face constitue une orientation typique : ainsi, lors des situations d'apprentissage en EPS, l'enseignant intervient pour redonner les consignes et répéter ses attentes. Enfin, le rite de réparation (RR): «lorsque les élèves ne suivent pas vos consignes et/ou redéfinissent la tâche proposée. Vous interagissez avec ces élèves pour obtenir leur adhésion». II s'agit ici de prévenir un événement qui est incompatible avec les valeurs sociales défendues et risque de perturber l'équilibre instauré préalablement. L'enseignant interagit pour contraindre l'élève à adhérer au but de la tâche. 
Pour chacun des trois rites, l'enseignant doit répondre à 13 questions (cf. annexe) relatives à une analyse réflexive post interaction.

Ce terme de rite nous intéresse car il définit un ensemble de règles, d'actes, d'arrangements inhérents à l'activité de l'enseignant. Nous mettrons également en exergue une typologie de profils professionnels.

L'analyse de contenu suppose de la part du chercheur de faire un " effort d'interprétation» Bardin (2007): 1) lecture profane des réponses avancées par les enseignants d'EPS, 2) lecture interprétative et analytique afin de faire émerger les marqueurs constitutifs de la construction de l'identité professionnelle dans la «zone de rencontre» lorsque l'enseignant interagit avec les élèves. Utiliser l'analyse de contenu pour faire vivre notre questionnaire, c'est mettre en avant que ce type d'analyse est avant tout une pratique inscrite dans une pragmatique (Ghiglione, Beauvois, Chabrol, Trognon, 1980, p.5) et qu'elle n'est qu'un processus d'investigation dans notre recherche. Cependant, ce type d'analyse permet d'établir une typologie textuelle (Robert, Bouillaguet, 2002). Elle se justifie par la nécessité de catégorisation mentale comme aide à la compréhension interne du corpus et constitue par là même les fondations de notre étude.

Dans un premier temps, nous avons mis en place un codage des éléments de réponse, puis dans un second temps, et à partir de ce codage, nous avons élaboré une catégorisation du corpus :

- le contexte : cette catégorie fait référence aux notions d'espace et de distance, plus particulièrement développées par Hall (1971),

- les techniques utilisées par l'enseignant pour faire apprendre : elles sont corporelles ou communicationnelles,

- la relation qui se construit lors des épisodes interactionnels et qui s'articule autour de la notion de rôle dans la classe,

- les attentes et les intentions didactiques dans la situation qui permettent à l'enseignant de construire un équilibre avec l'élève au sein de la situation d'interaction.

Ces quatre thèmes ont subi une transposition du domaine de la didactique à celui de la microsociologie, permettant le passage de la situation d'interaction de type sociologique à la situation d'enseignement, qui constitue une situation d'interaction particulière:

- l'idée de contexte conduit l'enseignant à parler de son espace d'intervention et de la distance qu'il conçoit entre lui et ses élèves,

- les techniques de transmission énumérées par les enseignants mettent en avant le rôle prioritaire du corps de l'enseignant et de sa place dans l'espace interactionnel, 
- la relation entre l'enseignant et les élèves se construit à partir d'un cadre établi entre les acteurs,

- les attentes et les intentions didactiques caractérisent des projets d'actions de l'enseignant significatifs de l'existence de motifs dans la situation proposée et d'exigences dans la tâche à réaliser.

Cette transposition donne lieu à l'identification de profils professionnels particuliers.

\section{2. L'observation de terrain}

Neuf enseignants ont été filmés lors de leçons d'EPS. Cette observation in situ a donné lieu d'une part à des notes de terrain (qui ont une fonction descriptive) et d'autre part à des analyses à priori (qui permettent d'attribuer une signification à ce qui a été décrit précédemment), à partir de la catégorisation du corpus de réponses issu du questionnaire. Elle a pour but de circonscrire la présence de «zones de rencontre », comme un espace probable de transmission de savoirs et a permis d'identifier par ailleurs des dimensions processuelles de la pratique professionnelle.

\section{3. L'étude de cas}

Elle rend compte d'un cas particulier, celui de "Thomas », le fil rouge de notre recherche (il a répondu au questionnaire et a été observé). Cette analyse intrinsèque et contextuelle nous permet de suivre l'usage particulier et intime des «zones de rencontre » construite par « Thomas » lors de ces rencontres avec les élèves :

- repérer en quoi les savoirs mis à l'étude deviennent des objets de transaction ;

- révéler les enjeux didactiques qui émergent de la « zone de rencontre » ;

- accéder par l'entretien d'auto confrontation (« Thomas » a été invité à commenter les traces filmées de son activité) à la dimension significative de son activité lors de la mise en place du processus interactionnel.

Nous avons suivi cet enseignant au cours de 2 leçons de lutte et de volley-ball (VB). Le recueil des données s'appuie sur deux modalités: une saisie vidéo des leçons et des entretiens d'auto-confrontation à l'issue des leçons. Nous retenons comme descripteurs les quatre catégories issues des questionnaires et utilisées lors de l'observation directe et participante. Ce choix exprime la volonté de conserver une cohérence à l'intérieur de notre cadre méthodologique afin de lui donner une singularité et un sens. Cette troisième focale s'organise à partir de trois niveaux de condensation des données.

- Le premier niveau de condensation des données correspond à une retranscription de ce que le chercheur lit des vidéos des trois leçons, ce niveau permet d'établir un découpage de la leçon au regard des catégories empruntées au premier type de données à savoir les questionnaires. 
- Le second niveau de condensation met en parallèle la transcription intégrale des leçons filmées avec le verbatim des entretiens d'auto confrontation. Enfin, le troisième niveau de condensation nous permettra de réorganiser et de synthétiser l'ensemble des données.

\section{Résultats}

La « zone de rencontre » $(Z R)$ présente des caractéristiques et des fonctions particulières.

3. 1. Le questionnaire

Les résultats montrent des caractéristiques précises structurées autour des rites d'interaction.

3. 1. 1. La Zone de Rencontre se construit dans un contexte particulier :

a) Dits sécuritaire en début de leçon (Rite de réparation, RP), l'enseignant maintient une distance comprise entre 2 et $3 \mathrm{~m}$ puis entre 3,50 et $7 \mathrm{~m}$ lors de la répétition des consignes organisationnelles (par exemple la mise en œuvre de l'échauffement)

b) Dits de proximité pendant la leçon, la distance est comprise entre $45 \mathrm{~cm}$ à $1,25 \mathrm{~m}$ pour l'activité lutte et de 2 à $3 \mathrm{~m}$ pour l'activité volley-ball. Ces résultats montrent que les enseignants semblent apprendre à organiser leur espace d'intervention qui tend à fluctuer selon la nature de l'APSA.

3. 1. 2. La Zone de Rencontre incite la mise en place de techniques pour négocier sa position et gérer sa classe. Les réponses déterminent l'existence d'un répertoire:

a) le corps est un outil pour rencontrer les élèves

b) le regard appuie les paroles et la gestuelle corporelle

c) la voix et son intonation sont des arguments de la communication.

3. 1. 3. La Zone de Rencontre existe quand la relation est mise en place : par exemple,

a) Lors de rite de présentation, les enseignants disent qu'ils ont un rôle à jouer et qu'il sera décisif, la rencontre est attendue et doit se construire.

b) Lors du rite de confirmation, les enseignants disent que leurs interventions sont significatives de la transmission de ce qu'il y a à apprendre.

c) Lors du rite de réparation, les enseignants disent qu'ils vont tenter de neutraliser la menace pour retrouver un équilibre. Pour eux, la négociation est interdite. 
3. 1. 4. La Zone de Rencontre fait émerger des attentes et des intentions didactiques.

Les enseignants parlent de la mise en place de cadres, d'espaces de réflexion et de dialogue pour permettre l'apprentissage. Par exemple :

a) Lors de rite de présentation, les réponses des enseignants portent sur les consignes organisationnelles, les objectifs de la leçon.

b) Lors du rite de confirmation, les réponses laissent entrevoir un enseignant qui livre le problème aux élèves, dirige leur activité et soutient leurs efforts,

c) Lors du rite de réparation, l'enseignant affirme qu'il négocie avec l'élève pour tenter de rétablir un équilibre entre ce qui est fait et ce qui est demandé.

De plus, les réponses des enseignants mettent en avant d'autres propriétés de la «zone de rencontre ». L'enseignant construit cet espace en articulant son projet personnel et professionnel au projet éducatif. A partir des intentions d'actions verbalisées dans les questionnaires, nous identifions une typologie de trois profils professionnels :

- l'enseignant « protecteur » correspond à $20 \%$ des réponses

- l'enseignant « transmetteur », $20 \%$

- l'enseignant « acteur », 60\%

Aussi, les réponses font émerger un processus dynamique de la rencontre construit à partir de trois logiques de ritualisation :

1) la logique sociale et spatiale construite autour des attitudes, des comportements et des projets de l'enseignant ;

2) la logique corporelle et personnelle constitue un véritable outil pour assumer le rôle du professeur d'EPS. En utilisant son corps, sa voix et son regard, l'enseignant donne à voir à ses élèves une certaine apparence ;

3) la logique professionnelle s'appuie sur un cadre institutionnel : la leçon, les objectifs à atteindre. 
Tableau I. Synthèse des traits caractéristiques des trois profils identifiés

\begin{tabular}{|c|c|c|c|}
\hline Profil & $\begin{array}{l}1 \\
\text { l'enseignant protecteur }\end{array}$ & $\begin{array}{l}2 \\
\text { l'enseignant transmetteur }\end{array}$ & $\begin{array}{l}3 \\
\text { l'enseignant } \\
\text { acteur }\end{array}$ \\
\hline $\begin{array}{l}\text { les } \\
\text { Caractéristiques }\end{array}$ & $\begin{array}{l}\text { angoissé, } \quad \text { stressé, } \\
\text { fusionnel }\end{array}$ & $\begin{array}{l}\text { efficace, recherche un } \\
\text { accomplissement personnel }\end{array}$ & $\begin{array}{l}\text { existe par et parmi les } \\
\text { élèves }\end{array}$ \\
\hline Son projet & $\begin{array}{l}\text { Eviter la rupture du contrat } \\
\text { didactique et éviter la } \\
\text { rupture avec les élèves. }\end{array}$ & $\begin{array}{l}\text { Faire réussir les élèves et } \\
\text { construire une estime de soi. }\end{array}$ & $\begin{array}{l}\text { Analyser le } \\
\text { fonctionnement des } \\
\text { élèves pour donner du } \\
\text { sens et agir } \\
\text { personnellement dans ce } \\
\text { processus. }\end{array}$ \\
\hline $\begin{array}{l}\text { les } \\
\text { Mises en œuvre }\end{array}$ & $\begin{array}{l}\text { Proximité spatiale avec les } \\
\text { élèves } \\
\text { Recherche incessante } \\
\text { d'une convivialité } \\
\text { relationnelle. } \\
\text { Communication basée sur } \\
\text { l'encouragement, la } \\
\text { complicité et l'écoute de } \\
\text { l'autre. }\end{array}$ & $\begin{array}{l}\text { Expliquer, organiser, faire } \\
\text { comprendre, verbaliser, } \\
\text { démontrer, réexpliquer, } \\
\text { questionner. } \\
\text { Permettre un réel } \\
\text { engagement de l'élève dans } \\
\text { la situation. }\end{array}$ & 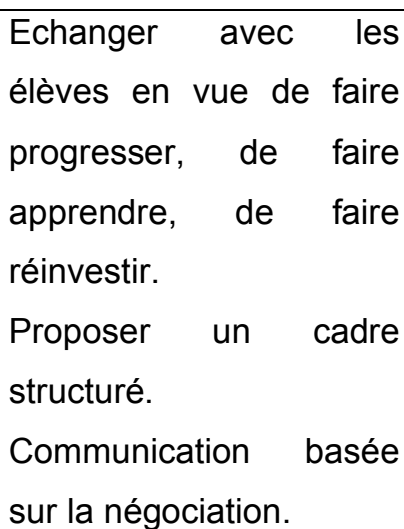 \\
\hline
\end{tabular}

\section{2. Les observations}

Elles permettent d'identifier un ensemble de phénomènes qui contraint l'enseignant à construire un cadre lors de ses rencontres avec les élèves. Nous nous plaçons ici au niveau de la macro structure sociale.

La « zone de rencontre » présente les caractéristiques suivantes :

- le contexte s'organise et se construit autour de la motricité des élèves :

L'enseignant doit parvenir à contrôler l'espace dans lequel il interagit avec ses élèves. Cet espace peut être petit et confiné comme la salle de tennis de table ou le dojo, au contraire il peut être grand comme le gymnase type « $C$ ». Cette organisation présente deux aspects particuliers : l'installation du matériel (sujet $n^{\circ} 2, s 2$ ), l'aménagement du milieu (s7) et la sécurisation des lieux (s9), ou la répartition des élèves (s4) afin que chacun d'entre eux puissent organiser sa motricité, apprendre (s2), comprendre les situations d'apprentissage (s5). Les déplacements sont nombreux, apparemment inspirés par l'organisation motrice des élèves et animés par des intentions précises de l'enseignant: expliquer la tâche (s5), organiser fonctionnellement la tâche (s7), contrecarrer la rupture du contrat (s4), démontrer un exercice (s5), complexifier une tâche (s5), réguler les 
apprentissages. Les rassemblements prennent deux formes, collectifs ou par petits groupes. Quelle que soit sa nature, le rassemblement semble être le prétexte à faire des bilans (s6), redonner des explications (s2), gérer le temps de la tâche (s3), mettre en place une nouvelle tâche (s7), évaluer (s4), délivrer les exigences de la tâche (s5), parler avec les élèves (s8).

- les techniques utilisées montrent la mise en place d'une conduite de rapprochement vers l'élève: l'enseignant souhaite conserver le contrôle de sa classe durant la leçon d'EPS. Pour se faire, il argumente ses choix d'exercices, donne le but des tâches, les moyens voire la technique à utiliser (s7, s8). L'appel du début de leçon sert souvent de prétexte à entrer dans la leçon et à affirmer son rôle auprès des élèves (s4), de même que présenter la leçon (s7), répondre aux questions des élèves et justifier ses réponses (s7), assurer la sécurité (s8), stimuler leur activité (s9). II s'agit aussi d'assumer son statut et de délivrer une culture qui s'appuie sur des savoirs de différentes natures (s1). L'enseignant se met en scène, les techniques sont diverses : l'enseignant peut guider l'élève dans ses apprentissages, dans l'organisation de sa motricité (s4), démontrer ce qu'il y a à faire et l'encourager (s2, s5, s8, s9), décrire les tâches (s3). Il peut aussi le questionner (s2), utiliser des fiches de travail (s5), parler avec eux de leur projet individuel ou collectif (s4) et partager leurs préoccupations (s5), les aider dans leurs choix, les laisser exprimer leurs doutes, leurs idées (s8), à partir de la motricité des élèves pour trouver des solutions efficaces au problème posé par la tâche $(\mathrm{s} 5, \mathrm{~s} 9)$. L'enseignant contrôle la motricité de ses élèves (s6). Pour cela, il peut aménager le milieu (s7), s'appuyer sur les rôles sociaux de l'activité support (s3, s7, s9). Son rôle va contraindre à l'élève à s'impliquer dans la leçon (s2), à répéter plusieurs fois la même action ( $\mathrm{s} 9$ ), à verbaliser sur ses actions, à donner ses solutions (s9) et à comprendre ce qu'il y a à faire ( $(s 6, s 9)$. Pour cela, l'enseignant d'EPS s'appuie sur des savoirs de différentes natures (s1, s2, s3, s8, s9), rappelle les exigences de la tâche (s7), observe les conduites de ses élèves, régule lorsqu'il y a nécessité (s5), signale aux élèves le décalage entre ce qui est fait et ce qu'il faut faire (s3). L'enseignant est amené à commenter ce qu'il observe (s6), ce qui le contraint à rappeler les objectifs de la leçon, les exigences de la tâche à accomplir (s6, s7), à donner les critères de réussite ou d'observation aux élèves ( $(5)$, à leur donner des repères spatiaux et temporels (s6, s8, s9), à utiliser des fiches de travail ou autres médias (s6). L'enseignant doit s'assurer que la tâche est comprise (s7) pour pouvoir délivrer les contenus d'enseignement (s7) et amener les élèves à réussir et à entrer dans une nouvelle tâche plus complexe ou plus simple (s9). La relation qu'il noue avec les élèves s'articule autour de la négociation, de la différenciation. Les attentes et les intentions 
didactiques s'orientent à partir de la recherche d'un équilibre entre ce qui est demandé et ce qui est fait. II construit un espace où les élèves agissent.

- les relations pour vivre son interaction didactique. Les enseignants d'EPS observés vivent leurs émotions comme un moteur de leur enseignement. Ils veulent être compris et écoutés, pour cela ils aménagent leurs espaces de travail et de rencontre avec l'élève et les encouragent (s3). Ils adoptent une différenciation pédagogique (s6) et s'accordent sur les attentes de l'autre (s5). L'élève devient un partenaire des apprentissages (s5). L'enseignant est amené à mettre en place une dévolution, une négociation implicite de la charge de travail et de l'investissement de l'élève dans la tâche (s5, s6, s7). La dévolution est définie comme « l'acte par lequel l'enseignant fait accepter à l'élève la responsabilité d'une situation d'apprentissage (a-didactique) ou d'un problème et accepte lui-même les conséquences de ce transfert »(Brousseau, 1988). L'enseignant comme l'élève sont acteurs dans la situation (s7) où la rencontre oblige l'action et la compréhension (s8). II s'agit de dépasser l'agir pour aller vers le plaisir de faire ou de faire faire (s8).

- les attentes et les intentions didactiques : tout ce qui est mis en place dans la leçon, lors des rencontres entre l'enseignant et ses élèves montre que la situation d'interaction n'existe qu'à travers un équilibre instable qui doit se construire dans la zone interactionnelle. Révéler son cadre, c'est tenter de le faire respecter (s2, s4), c'est présenter ses attentes et les faire comprendre aux élèves (s4). II s'agit pour l'enseignant d'être clair et de trouver les mots qui ont du sens pour les élèves (s4), de s'appuyer sur les représentations de ces derniers afin de leur proposer les contenus en cohérence avec leurs motifs (s2). Pour l'élève, cela signifie acquérir des compétences pour pouvoir progresser, percevoir les exigences, accepter les contraintes et trouver les solutions au problème posé (s8). II s'agira donc d'écouter, de comprendre et d'estimer ce qu'il faut faire. L'enseignant doit faciliter l'engagement de l'élève dans la situation d'apprentissage (s1) afin que celui-ci puisse acquérir des habitudes de travail (s3), des méthodes (s8), des connaissances (s8), des routines et des techniques (s5) pour devenir efficace dans l'activité support. L'enseignant d'EPS doit apprendre à ses élèves à écouter, à parler, à organiser son travail (s6) seul ou collectivement (s7) pour trouver des solutions motrices (s7), à réfléchir et à s'évaluer (s6) pour réinvestir ses acquis ailleurs, à observer l'autre (s7) pour valider son niveau de maîtrise (s8), à dépasser la simple imitation (s9) pour comprendre son action et devenir acteur de ses apprentissages (s9). Ainsi, l'enseignant à travers son projet d'enseignement aide l'élève à se rapprocher de son projet personnel (s8), à utiliser et à gérer des techniques motrices. 
En résumé, il est possible de dégager de cette analyse des particularités. La « zone de rencontre » devient: le lieu d'analyse des besoins des formés; une véritable situation d'apprentissage pour les élèves ; révélatrice de la volonté d'une transmission des savoirs ; enfin, un laboratoire d'analyse réflexive pour les enseignants.

\section{3. L'étude de cas}

3. 3. 1. Le premier niveau de condensation fait apparaître :

Ce qu'il y a faire apprendre dans les APSA support : par exemple pour les leçons filmées de lutte (Tableau II et III) ainsi que les moyens pour y parvenir (dans les 4 leçons d'EPS) Tableau II. Ce qu'il y a à apprendre dans les leçons $n^{\circ} 4$ et $n^{\circ} 5$ de lutte

\begin{tabular}{|l|}
\hline Mettre en place des surfaces de travail sécurisées \\
\hline Apprendre des techniques de contrôle \\
\hline Enoncer des règles de sécurité \\
\hline Incorporer les rôles sociaux \\
\hline Comprendre la notion de déséquilibre des corps \\
\hline Appréhender le poids de son adversaire \\
\hline Contrôler le corps de l'autre \\
\hline Donner une direction à son corps \\
\hline Arbitrer un combat \\
\hline
\end{tabular}

Tableau III. Les moyens pour y parvenir communs aux leçons filmées d'EPS de Thomas

\begin{tabular}{|l|}
\hline $\begin{array}{l}\text { Objets communs à la leçon d'Education Physique } \\
\text { et Sportive, LL4, LL5, VBL4 }\end{array}$ \\
\hline L'habitus de l'échauffement \\
\hline Proposer et imposer des objectifs \\
\hline Démontrer des techniques pour faire apprendre \\
\hline Manipuler des corps pour faire sentir \\
\hline Gérer le temps des apprentissages \\
\hline Observer pour organiser la motricité des élèves \\
\hline $\begin{array}{l}\text { Commenter les actions des élèves pour faire intégrer } \\
\text { des savoirs }\end{array}$ \\
\hline $\begin{array}{l}\text { Répéter les consignes pour permettre aux élèves } \\
\text { d'intérioriser et de comprendre les savoirs enseignés }\end{array}$ \\
\hline Encourager pour motiver l'activité des élèves \\
\hline Se rassembler pour faire un bilan \\
\hline
\end{tabular}


3. 3. 2. Le second niveau de condensation permet de présenter la démarche d'enseignement de Thomas ainsi qu'une modélisation de son activité. Pour ce faire, nous empruntons à Schütz (1987) la notion de « d'action » "au sens de conduite humaine basée sur un projet pré-conçu. À cette notion de projet, sont associées celle de motifs ». II s'agit donc d'identifier :

- la nature des motifs " en vue de », qui se réfèrent à une fin en vertu de laquelle une action est faite, que nous avons transposée en motif « en vue », à savoir les effets que Thomas vise in situ par ses actes et ses paroles (Gal-Petitfaux, Cizeron, 2006).

Tableau IV. Les motifs « en vue »

\begin{tabular}{l}
\hline Les motifs « en vue » \\
\hline S'assurer que la situation est comprise et acceptée: \\
Thomas répète les consignes durant toute la durée \\
de la situation : à la classe, au groupe d'élèves, à \\
l'élève. \\
\hline $\begin{array}{l}\text { Donner des indicateurs d'auto observation, des } \\
\text { critères de réussite. }\end{array}$ \\
\hline
\end{tabular}

- des motifs « parce que » qui réfèrent plutôt à l'intégration d'un réseau d'éléments passés, tel que soulevé par des lectures d'événements (lorsque "Thomas » examine les vidéos) transposés en motif "perçu» (la signification que donne Thomas à ses actes lorsqu'il se regarde).

Tableau V. Les motifs «perçus »

\begin{tabular}{|l|}
\hline Les motifs « perçus » \\
\hline S'assurer que ses choix sont acceptés par les élèves \\
\hline Faire accepter aux élèves le cadre institutionnel \\
\hline Transmettre les savoirs appropriés aux besoins des \\
élèves \\
\hline $\begin{array}{l}\text { Acquérir des habitudes de travail. } \\
\text { Devenir acteur de ses apprentissages }\end{array}$ \\
\hline
\end{tabular}


3. 3. 3. Le troisième niveau de condensation (la micro structure interactionnelle).

Les savoirs extraits de ce dernier niveau d'analyse s'organisent selon deux blocs (Chevallard, 1997) : la praxie ou pratique reliant une tâche à une technique ; on l'identifie à un savoir-faire et la technique permet de réaliser la tâche d'une part. D'autre part, le logos ou discours raisonné reliant une technologie à une théorie dominante. On l'identifie à une théorie des savoirs sur le faire.

Tableau. VI La praxie

\begin{tabular}{|l|}
\hline Tâche - Technique \\
\hline Tâche : immobiliser l'adversaire (finale costale et \\
latérale) \\
\hline Technique : \\
- construire des zones de contact et de contrôle \\
$\quad$ sur le corps de l'adversaire \\
- appréhender le rôle de la tête \\
\hline
\end{tabular}

Tableau VII. Le logos

\begin{tabular}{|l|}
\hline Technologie - Théorie \\
\hline Technologie : \\
- imposer un état corporel à autrui \\
- contrôler certaines zones du corps de \\
l'adversaire par des saisies et des points \\
d'appuis appropriés et utiliser la posture \\
adéquate \\
\hline Théorie : la biomécanique et le pôle affectif \\
\hline
\end{tabular}

Pour extraire les savoirs nous postulons qu'ils sont transmis à l'élève à partir des informations délivrées par l'enseignant dans la « zone de rencontre ».

Nous nous référons aux catégories issues des travaux de Le Boterf (1997) qui sont exposées dans le tableau VIII. 
Tableau VIII. Exemples de savoirs transmis dans les leçons de lutte et de volley-ball

\begin{tabular}{|c|c|}
\hline \multicolumn{2}{|l|}{ Les Savoirs } \\
\hline savoir théorique : savoir comprendre & $\begin{array}{l}\text { Comprendre l'organisation des combats et } \\
\text { respecter les consignes de sécurité }\end{array}$ \\
\hline savoir d'environnement : savoir s'adapter & $\begin{array}{l}\text { Réaliser des saisies différenciées sur } \\
\text { l'adversaire: tirer avec une main, pousser } \\
\text { avec l'autre, soulever avec une main et } \\
\text { abaisser avec l'autre. }\end{array}$ \\
\hline $\begin{array}{l}\text { savoir procédural : savoir comment } \\
\text { procéder }\end{array}$ & $\begin{array}{l}\text { Isoler une technique puis la mettre en œuvre } \\
\text { sur le corps de son adversaire. }\end{array}$ \\
\hline savoir faire opérationnel : savoir opérer & $\begin{array}{l}\text { Se mettre en activité et reproduire des } \\
\text { techniques d'échauffement apprises lors des } \\
\text { leçons précédentes. }\end{array}$ \\
\hline $\begin{array}{l}\text { savoir faire expérientiel et existentiel: } \\
\text { savoir y faire }\end{array}$ & $\begin{array}{l}\text { Verbaliser mes actions motrices et ma façon } \\
\text { de fonctionner. }\end{array}$ \\
\hline savoir faire social : savoir se conduire & Aider à ranger le matériel. \\
\hline $\begin{array}{l}\text { savoir faire cognitif : savoir traiter } \\
\text { l'information }\end{array}$ & $\begin{array}{l}\text { Ecouter et comprendre ce qu'il y a à faire } \\
\text { dans la tâche présentée. }\end{array}$ \\
\hline
\end{tabular}

\section{Discussion}

L'ensemble des résultats permet de comprendre l'activité de l'enseignant dans la « zone de rencontre », à partir des trois strates d'analyse (Pastré, Samurçay, Bouthier, 1995) :

\section{1. La macro structure sociale}

Elle s'imprègne du cadre institutionnel, en porte les valeurs et s'articule autour de rites d'interaction. Les espaces observés et analysés montrent une réelle perspective d'apprentissage à travers l'organisation de la motricité des élèves. L'enseignant apprend à contrôler ces espaces, à contrôler sa classe et sa place. Ces constructions sociales laissent entrevoir des conceptions d'enseignement comme l'échauffement, véritable habitus corporel de l'EPS, ou la démonstration, la verbalisation ou encore l'observationmais aussi des savoirs à transmettre de nature particulière : des techniques, des connaissances et des sensations.

\section{2. La méso structure interactionnelle}

Elle permet la construction d'un équilibre et d'un partage des responsabilités entre l'enseignant et l'élève.

La présence de cadres fait émerger des structures de différentes natures: 
a) une structure relationnelle où l'enseignant anticipe les intentions qui orientent les actions des élèves,

b) une structure conversationnelle : la zone de rencontre est un lieu de communication verbale et non verbale, un lieu de dialogue et de négociation, un lieu de transmission,

c) une structure situationnelle : la rencontre a lieu dans la situation d'apprentissage lorsque celle-ci devient la situation d'interaction, l'enseignant met en scène le contrat didactique....

d) une structure personnelle : l'enseignant appartient à la situation, il met en place des techniques corporelles, un code de fonctionnement.

\section{3. La micro structure interactionnelle}

Elle fait émerger une construction et un échange des savoirs. Ces derniers sont régulièrement mis en œuvre pour résoudre des problèmes quotidiens et révèlent chez l'enseignant une pensée d'exploration et de construction, une réflexion en action, un agir réflexif (Schön, 1983). En effet, les acteurs sont en mesure d'élaborer conjointement des actions qui en retour contribuent à la création de savoirs. Au-delà, l'enseignant apprend à gérer ses émotions dans l'interaction. Cette particularité fait émerger une facette humaine pas toujours prises en compte dans les recherches en didactique. En effet, nous avons postulé la réciprocité mutuelle dans l'interaction. A cette réciprocité s'ajoute, à la manière de Goffman (1973) la mise en scène des acteurs dans l'interaction.

- L'enseignant doit apprendre à contrôler les affres et les vertiges de cette mise en scène, il doit apprendre à jouer un rôle et à l'accepter.

- L'enseignant d'EPS va apprendre à s'impliquer corporellement dans la relation : « Le corps se trouve à la charnière des concepts apparemment antagonistes : la nature et la culture, l'homme et la société, le privé et la public, la raison et l'émotion, l'intelligence abstraite et l'intelligence concrète » (Vygotski, 2003). L'enseignant apprend à adopter la bonne posture dans la «zone de rencontre » et à lire les difficultés des élèves à travers leur corps et la façon dont il l'utilise pour répondre au problème que pose la situation d'apprentissage, la situation d'interaction. Les trois niveaux d'interrogation ainsi révélés laissent entrevoir une multi référencialité de réponses et l'existence d'un système qui s'organise in situ au moment de l'interaction, dans la « zone de rencontre ».

L'interaction constitue un véritable processus circulaire (Baeza, 2001), socialement situé. La «zone de rencontre » est à construire par les acteurs et c'est à l'intérieur de celle-ci que va se produire la co-construction des savoirs, c'est-à-dire la transmission par l'enseignant et l'appropriation par les élèves. Ce « face à face » s'apparente à un véritable 
partage de significations, choisies par les acteurs et construites mutuellement dans la situation d'interaction.

Notre cadre d'analyse postulait une certaine complexité avec laquelle nous avons dû jouer pour montrer que derrière le processus de «transmission » se cache d'autres phénomènes telle que celui de la construction de l'identité professionnelle, corporelle et personnelle. Ces processus se construisent en situation et sont étroitement liés aux circonstances locales et sociales.

\section{Bibliographie}

Amade-Escot, C. (1996). L'observation des activités didactiques en Education Physique et Sportive : aspects méthodologiques. Impulsions, 1. 75-98.

Amade-Escot, C. (2002). De l'usage des théories de l'enseignant, questions soulevées par l'étude des contrats didactiques en éducation physique. In A. Mercier, G. Lemoyne, A. Rouchier (eds). Le génie didactique : usages et mésusages des théories de l'enseignement. Bruxelles: De Boeck Université. (pp. 23-41). Bruxelles : De Boeck.

Baeza, N. (2001). Morceaux choisis: les interactions en EPS. Interagir avec ses élèves pour se construire en tant qu'enseignant. Mémoire pour l'obtention du DEA. Paris : Université Paris XI.

Bardin, L. (1997). L'analyse de contenu. Paris : PUF.

Bouthier, D., Pastré, P., \& Samurçay, R. (1995). Le développement des compétences. Analyse du travail et didactique professionnelle. Education Permanente, 123-2. 149.

Brousseau, G. (1978). L'observation des activités didactiques. Revue Française de Pédagogie, 45, 130-140.

Brousseau, G. (1988). Le contrat didactique: le milieu. Recherches en Didactique des Mathématiques, (9), 3, 309-336.

Chevallard, Y. (1997). Familière et problématique, la figure du professeur. Recherche en didactique des mathématiques, 17/2, 17-54.

Delvolvé, N., \& Margot, A. (1996). Le travail de l'enseignant d'un point de vue de l'ergonomie. Psychologie et Education, 44, 43-54.

Fabre, M. (1994). Penser la formation. Paris : PUF. 
Gal-Petitfaux, N., \& Cizeron, M. (2006). Motifs « cachés » et motifs « apparents » du dialogue professeur-élèves lors des bilans de leçon : étude des cas d'enseignants novices en Education Physique. eJRIEPS, 10, juillet, 21-33.

Ghiglione, R., Beauvois, JL., Chabrol, C., \& Trognon, L. (1980). Manuel d'analyse de contenu. Paris : Armand Colin.

Goffman, E. (1973). La mise en scène de la vie quotidienne. Paris : Minuit.

Goffman, E. (1974). Les rites d'interaction. Paris : Minuit

Hall, E. T. (1966-71). The Hidden Dimension, New York. Traduction française: La Dimension cachée. Paris : Seuil.

Jonnaert, P. (1996). Dévolution versus contre dévolution! Un tandem incontournable pour le Contrat didactique. In C. Raisky \& M. Caillot (Eds.), Au-delà des didactiques, le didactique. Débat autour de concepts fédérateurs (pp 115-144). Bruxelles : De Boeck Université.

Le Boterf, G. (1997). De la compétence à la navigation professionnelle. Paris : Les Edition d'Organisation.

Marcellini A., \& Miliani, M. (1999). Lecture de Goffman. Corps et Culture, 4. Corps, Sport et Rites, [En ligne], mis en ligne le 24 septembre 2007.

Robert André, D., \& Bouillaguet, A. (2002). L'analyse de contenu. Paris : PUF.

Pastré, P., Samurçay,, R, Bouthier, D. (1995). Le développement des compétences. Analyse du travail et didactique professionnelle. Education Permanente, 123.

Pastré, P., Mayen, P., \& Vergnaud, G. (2006). La didactique professionnelle. Revue Française de Pédagogie,154, 145-198.

Samurçay, R., \& Pastré, P. (1998). L'ergonomie et la didactique, l'émergence d'un nouveau champ de recherche, la didactique professionnelle. Colloque : Recherche et ergonomie. Toulouse. (9, 10, 11 février 1998).

Schön, D. (1983, 1994). Le praticien réflexif. A la recherche du savoir caché dans l'agir professionnel. Montréal : Logiques.

Schütz, A. (1987). Le chercheur et le quotidien. Paris : Méridiens Klingsieck.

Vygotski, L.S. (2003). Conscience, inconscience, émotions. Paris : La Dispute. 


\section{Annexes}

Annexe $\mathrm{n}^{\circ} 1$ : Le questionnaire

-Quelle est la distance qui vous sépare de vos élèves ?

-Quelle perception avez-vous de votre espace de rencontre entre vous et vos élèves ?

-Quels types d'interventions verbales avez- vous avec vos élèves ?

-Quels sentiments ressentez-vous à ce moment là ?

-Quels types d'interventions avez-vous dans ces moments là ?

Q Quelles sont vos intentions dans ces moments là ?

Quel rôle voulez-vous jouer dans ces moments là ?

Q Quelle mise en scène adoptez-vous avec votre corps ?

-Comment utilisez-vous votre regard pendant ces moments ?

- Comment utilisez-vous votre voix pendant ces moments là ?

-Comment faites-vous pour capter leur attention?

Q Quels contacts voulez-vous établir avec vos élèves pendant ces moments là ?

-Qu'attendez-vous de vos élèves pendant ces moments là ? 